\title{
Metallic-Type Oscillatory Interlayer Exchange Coupling across an Epitaxial FeSi Spacer
}

\author{
R. R. Gareev, D. E. Bürgler,* M. Buchmeier, D. Olligs, R. Schreiber, and P. Grünberg \\ Institut für Festkörperforschung, Forschungszentrum Jülich GmbH, D-52425 Jülich, Germany
}

(Received 2 November 2000; published 21 September 2001)

\begin{abstract}
We study interlayer exchange coupling in epitaxial $\mathrm{Fe} / \mathrm{Fe}_{0.56} \mathrm{Si}_{0.44} / \mathrm{Fe}$ trilayers. Iron-silicide spacers with high structural and compositional homogeneity for thicknesses up to $34 \AA$ are grown by coevaporation from two electron-beam sources. The coupling strength oscillates with spacer thickness for temperatures from 20 to $300 \mathrm{~K}$ with two antiferromagnetic maxima at 12 and $26 \AA$, and it clearly increases with decreasing temperature down to $80 \mathrm{~K}$. We conclude that the coupling across ordered $\mathrm{Fe}_{1-x} \mathrm{Si}_{x}$ $(x \approx 0.5)$ is described by the conventional theory of interlayer coupling across metallic spacers.
\end{abstract}

PACS numbers: 75.70.-i, 73.61.At, 75.30.Et

Since the discovery of the antiferromagnetic (AF) exchange coupling between ferromagnetic layers separated by a nonmagnetic metal spacer [1], this phenomenon has been observed for a wide range of metallic spacer materials [2]. A further increased interest in this field has been stimulated by the finding of AF coupling through nonmetallic amorphous $\mathrm{Si}[3]$ and strong AF coupling across iron-silicide spacers [4]. Precise structural measurements proved that metallic iron silicides with an epitaxially stabilized cubic $\mathrm{CsCl}$ (B2) structure are preferably formed in a spacer as a result of a strong interdiffusion at the $\mathrm{Si} / \mathrm{Fe}$ interfaces [5-7]. It is now well established that the interlayer coupling through metallic spacers is connected with indirect RKKY-type exchange and that it oscillates from ferromagnetic (FM) to AF as a function of spacer thickness [8]. Hence, an oscillatory behavior of the coupling through metallic iron-silicide spacers is expected. However, in spite of intense research dealing with coupling phenomena across crystalline iron silicides no evidence of oscillatory exchange coupling was observed so far for these materials. Recently, de Vries et al. [9] reported an exponential decrease of the coupling strength with spacer thickness mediated by metallic FeSi spacers in epitaxial $\mathrm{Fe} / \mathrm{FeSi} / \mathrm{Fe}$ trilayers and concluded to have found a new type of interlayer coupling. Moreover, a strong enhancement of the coupling strength through highly resistive $\mathrm{FeSi}$ with more than $80 \%$ content of $\mathrm{Si}$ in $\mathrm{Fe} / \mathrm{FeSi}$ superlattices [10] demonstrate that the mechanism of exchange coupling through iron silicides is still far from being understood and therefore needs a further investigation.

The essential demand for a proper investigation of oscillatory interlayer coupling through binary compounds is a high degree of structural and compositional homogeneity of the spacer layer for all thicknesses of interest. However, epitaxial growth of $\mathrm{Fe} / \mathrm{FeSi} / \mathrm{Fe}$ trilayers has previously been observed only for spacer thicknesses $d_{\mathrm{FeSi}} \leq 20 \AA$, and additionally iron silicides of different composition and structure were usually formed by interdiffusion at the interfaces [6,9]. Furthermore, $\mathrm{Fe} / \mathrm{Si}$ and $\mathrm{Si} / \mathrm{Fe}$ interfaces appeared to be inequivalent with respect to the iron-silicide formation [11]. In this work we employ codeposition of $\mathrm{Fe}$ and $\mathrm{Si}$ to obtain more homogeneous $\mathrm{Fe}_{1-x} \mathrm{Si}_{x}$ spacers with well defined composition $x$. According to Ref. [12] the interdiffusion at $\mathrm{Fe} / \mathrm{FeSi}$ interfaces is strongly suppressed in comparison to $\mathrm{Fe} / \mathrm{Si}$ interfaces. Hence we prepare epitaxial $\mathrm{Fe} / \mathrm{Fe}_{1-x} \mathrm{Si}_{x} / \mathrm{Fe}$ trilayers with a nominal composition of the spacer layer close to $x=0.5$ of the stoichiometric $\mathrm{B} 2$ phase with $\mathrm{CsCl}$ structure or the $\mathrm{B} 20(\epsilon-\mathrm{FeSi})$ phase.

Epitaxial $\mathrm{Fe} / \mathrm{FeSi}$-wedge/Fe sandwiches are grown in an ultrahigh vacuum multichamber molecular beam epitaxy system by e-gun evaporation onto $\operatorname{GaAs}(100) /$ $\mathrm{Fe}(1 \mathrm{~nm}) / \operatorname{Ag}(150 \mathrm{~nm})$ substrate-buffer systems described elsewhere $[13,14]$. In order to minimize segregation of $\mathrm{Ag}$ [15] the first $4 \mathrm{ML}$ of the bottom 50 - $\AA$-thick Fe layer are grown at room temperature (RT) and the remaining at $473 \mathrm{~K}$. All thicknesses and deposition rates are controlled by calibrated quartz-crystal monitors. The wedge-shaped FeSi spacers are prepared at $473 \mathrm{~K}$, too. Two separate electron guns are used to codeposit $\mathrm{Fe}$ and $\mathrm{Si}$ at equal atomic flux to yield $\mathrm{Fe}_{0.5} \mathrm{Si}_{0.5}$. The thickness of the alloy layer is then given by

$$
d_{\mathrm{FeSi}}=1.06 d_{\mathrm{Si}}=0.67\left(d_{\mathrm{Fe}}+d_{\mathrm{Si}}\right),
$$

where $d_{\mathrm{Fe}}$ and $d_{\mathrm{Si}}$ are the quartz-crystal readings for $\mathrm{Fe}$ and $\mathrm{Si}$, respectively [9]. The spacer thickness $d_{\mathrm{FeSi}}$ varies along the wedge linearly from zero to $34 \AA\left(d_{\mathrm{Fe}}+d_{\mathrm{Si}}=\right.$ $0-50 \AA$ ). Finally, an upper $50-\AA$-thick Fe layer and a 500- $\AA$-thick ZnS coating are deposited at RT.

The composition and the structure of the $\mathrm{Fe} / \mathrm{FeSi} / \mathrm{Fe}$ trilayers are verified in situ by Auger electron spectroscopy (AES) and low-energy electron diffraction (LEED), respectively. A well-defined LEED (00) spot (at $75 \mathrm{eV}$ electron energy) could be observed for both Fe layers and for the whole range of $d_{\mathrm{FeSi}}$ indicating epitaxial growth. The spacer composition is calculated from $\mathrm{Fe}$ and $\mathrm{Si}$ deposition rates as well as from Auger spectra. In Fig. 1 a typical dependence of the nominal FeSi composition on spacer thickness is shown (open symbols). The spacer composition is also calculated from the Auger spectral intensities of $\mathrm{Fe}(703 \mathrm{eV})$ and $\mathrm{Si}(92 \mathrm{eV})$ lines taking into account the contribution of the bottom Fe layer to the Fe Auger signal due to the finite information depth of AES (filled symbols in Fig. 1). Both methods to determine the Si content agree well and confirm the homogeneous composition 


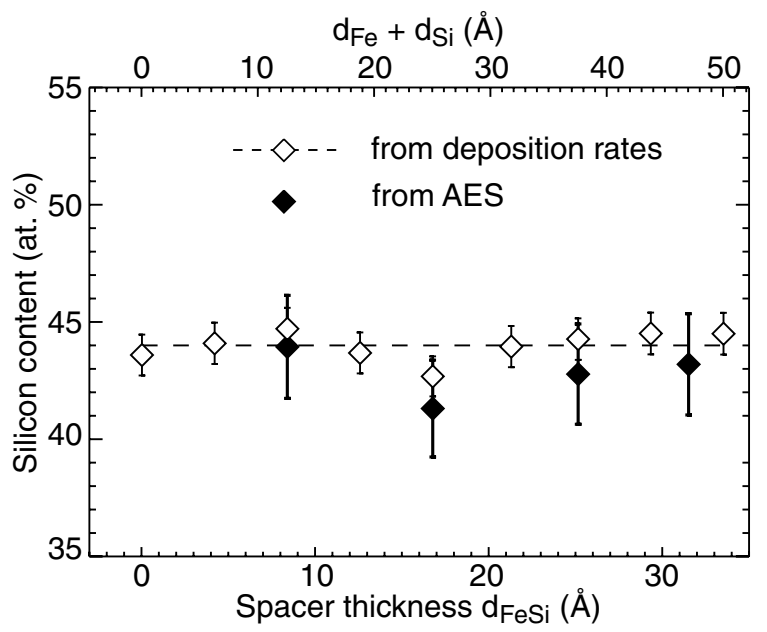

FIG. 1. Si content $x$ of a wedge-shaped $\mathrm{Fe}_{0.56} \mathrm{Si}_{0.44}$ spacer layer as a function of spacer thickness. Open symbols: determined from the deposition rates measured by means of two quartzcrystal monitors. Filled symbols: calculated from AES intensity ratios measured at different positions along the wedge taking into account contributions from the bottom Fe layer (inelastic mean free paths of the Auger electrons: $\lambda_{\mathrm{Si}}=2 \mathrm{ML}$ and $\lambda_{\mathrm{Fe}}=$ $5 \mathrm{ML}$ [16]). Abscissa: $d_{\mathrm{Fe}}+d_{\mathrm{Si}}$ is the sum of the nominally deposited thicknesses of $\mathrm{Fe}$ and $\mathrm{Si}$, and $d_{\mathrm{FeSi}}$ is the thickness of the resulting alloy layer according to Eq. (1).

of the spacer for $d_{\mathrm{FeSi}}$ up to $34 \AA$. The deposition rates yield an average nominal composition $x=0.44 \pm 0.01$ (dashed line in Fig. 1). Hence, we can exclude the formation of $\mathrm{Fe}_{3} \mathrm{Si}$. Spacers intentionally prepared with lower $\mathrm{Si}$ content $(x \approx 0.36)$ did not show $\mathrm{AF}$ or $90^{\circ}$ coupling. We explain this with the onset of FM order [17].

The magnetic properties are checked by longitudinal magneto-optic Kerr effect (MOKE) measurements with the external field applied in the sample plane. The MOKE setup based on an optical cryostat allows temperature dependent measurements in the range from 20 to $300 \mathrm{~K}$ and has previously been described in Ref. [18].

Easy-axis MOKE hysteresis loops for the spacer thicknesses $d_{\mathrm{FeSi}}=12$ and $26 \AA$ taken at 20 and $300 \mathrm{~K}$ are shown in Fig. 2. The asymmetries and the peaks around zero field are caused by second-order MOKE effects and temperature-dependent relative contributions of the two $\mathrm{Fe}$ layers to the MOKE signal. Our analysis given below is based on switching fields and on the presence and absence of remanent magnetization and hence is not influenced by these effects $[18,19]$.

For very large in-plane, fourfold anisotropy $K$ or negligible $K$ the saturation field can be used as a measure of the coupling. We measured the anisotropy field $H_{K}$ of our sample in the FM coupled regions (i.e., at thicknesses $d_{\mathrm{FeSi}}=3-10,16-20,32 \AA$ ) from hard-axis MOKE loops and found $K=H_{K} M_{S} / 2=(4.5 \pm 0.4) \times 10^{4} \mathrm{~J} / \mathrm{m}^{3}$ in good agreement with the Fe bulk anisotropy constant. $M_{S}=1.714 \times 10^{6} \mathrm{~A} / \mathrm{m}$ is the saturation magnetization of Fe. If $K t$ is of the same order of magnitude as the coupling $J$ as it will turn out to be the case for our

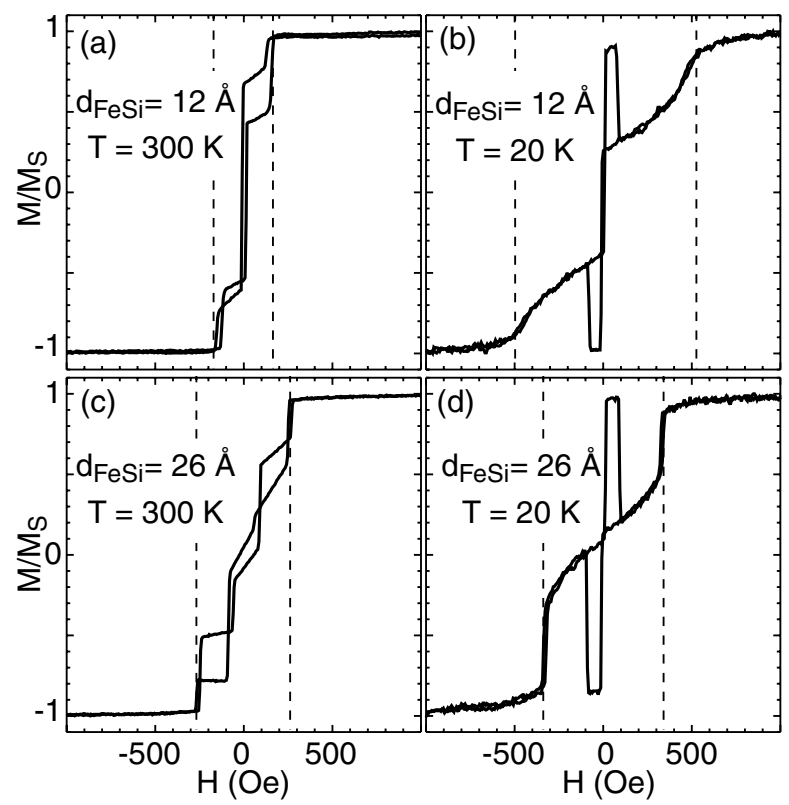

FIG. 2. Longitudinal MOKE hysteresis loops of a wedge-type $\mathrm{Fe}(5 \mathrm{~nm}) / \mathrm{Fe}_{0.56} \mathrm{Si}_{0.44}\left(d_{\mathrm{FeSi}}\right) / \mathrm{Fe}(5 \mathrm{~nm})$ trilayer for (a) $d_{\mathrm{FeSi}}=$ $12 \AA$ and $300 \mathrm{~K}$, (b) $d_{\mathrm{FeSi}}=12 \AA$ and $20 \mathrm{~K}$, (c) $d_{\mathrm{FeSi}}=26 \AA$ and $300 \mathrm{~K}$, and (d) $d_{\mathrm{FeSi}}=26 \AA$ and $20 \mathrm{~K}$. Dashed lines indicate the switching fields $H_{S}$.

samples, then we cannot derive an analytical relation between the saturation field and the coupling strength. However, we can define for all our MOKE loops a switching field $H_{S}$ (see, e.g., dashed lines in Fig. 2) where the magnetizations of the Fe films jump either to saturation or to a symmetric alignment with both magnetizations slightly deviating from the field axis. Considering the anisotropy $K$, bilinear $\left(J_{1}\right)$ and biquadratic $\left(J_{2}\right)$ coupling, and the external field $H$ hysteresis loops can be obtained by minimizing for each value of $H$ the total areal energy density of the system,

$$
\begin{aligned}
E= & -H M_{S} t\left[\cos \left(\vartheta_{1}\right)+\cos \left(\vartheta_{2}\right)\right] \\
& +\frac{K t}{4}\left[\sin ^{2}\left(2 \vartheta_{1}\right)+\sin ^{2}\left(2 \vartheta_{2}\right)\right] \\
& -J_{1} \cos \left(\vartheta_{1}-\vartheta_{2}\right)-J_{2} \cos ^{2}\left(\vartheta_{1}-\vartheta_{2}\right),
\end{aligned}
$$

with respect to the orientation of the magnetizations of the two films given by the angles $\vartheta_{1}$ and $\vartheta_{2}$ which are measured relative to the field axis. The magnetization $M(H)$ is then given by

$$
M(H)=\frac{M_{S}}{2}\left[\cos \left(\vartheta_{1}^{\min }(H)\right)+\cos \left(\vartheta_{2}^{\min }(H)\right)\right] .
$$

The Fe films are taken to be of equal thickness $t$. Varying the coupling constants $J_{1}$ and $J_{2}$ we find from the simulated hysteresis loops that the switching field $H_{S}$ is in good approximation proportional to the total coupling $J=J_{1}+J_{2}$.

The dependence of $H_{S}$ on the spacer thickness is measured for different temperatures ranging from 20 to $300 \mathrm{~K}$. 
Figure 3 shows the resulting coupling curves taken at 20 and $300 \mathrm{~K}$. We observe an oscillatory behavior of the coupling versus spacer thickness in the whole temperature range. Two distinct regions with clear coupling maxima are found near $d_{\mathrm{FeSi}}=12$ and $26 \AA$. The positions of the maxima are not dependent on temperature. The oscillations are more pronounced at higher temperatures where the hysteresis loops suggest a FM coupled thickness range $\left(d_{\mathrm{FeSi}}=16-20 \AA\right)$ between the two coupling maxima. We note that these data represent the first observation of oscillatory interlayer coupling across an FeSi spacer layer. The positions of the first peak matches the results of de Vries et al. [9], but the second peak contradicts their exponential decay of the coupling. We relate this discrepancy to (i) the more homogeneous spacer of our samples and (ii) the larger epitaxial spacer thickness range accessible in our experiments. Both advantages arise from the preparation of the FeSi spacer by coevaporation instead of interdiffusion. A mechanism for how structural disorder in a metallic spacer can lead to an exponential thickness dependence of RKKY-type interlayer coupling is described in Ref. [20]. Obviously, only the fact that $d_{\mathrm{FeSi}}$ is not limited to values smaller than $20 \AA\left(d_{\mathrm{Fe}}+d_{\mathrm{Si}}=30 \AA\right)$ - as it is the case in Ref. [9]-allows one to observe the oscillatory behavior.

The temperature dependence of the coupling at the first $J^{(1)}$ and second $J^{(2)}$ coupling maximum is shown in Fig. 4. We have determined the total coupling strength $J=J_{1}+$ $J_{2}$ by simulating hysteresis loops that reproduce the measured switching fields. Note that the values of $J$ are of the same order of magnitude as $K t$. An unequivocal separation of $J_{1}$ and $J_{2}$ is possible for MOKE loops that exhibit three plateaus. An example is Fig. 2(c) for which we obtain $J_{1}=-0.14 \mathrm{~mJ} / \mathrm{m}^{2}$ and $J_{2}=-0.07 \mathrm{~mJ} / \mathrm{m}^{2}$. These coupling constants are in the typical range obtained for many other metallic spacer layers. $J^{(1)}$ exhibits a monotonic increase upon cooling, whereas $J^{(2)}$ first increases down to

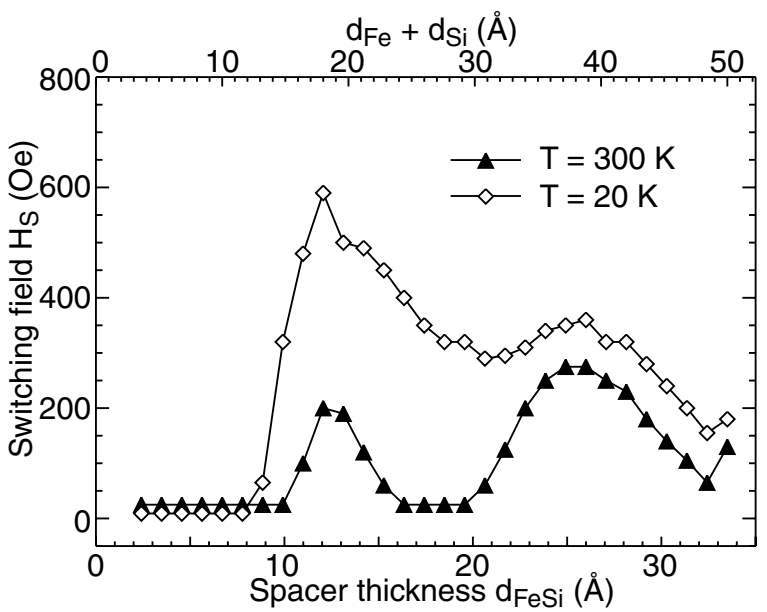

FIG. 3. Switching field $H_{S}$ versus spacer thickness $d_{\mathrm{FeSi}}$ of a wedge-type $\mathrm{Fe}(5 \mathrm{~nm}) / \mathrm{Fe}_{0.56} \mathrm{Si}_{0.44}\left(d_{\mathrm{FeSi}}\right) / \mathrm{Fe}(5 \mathrm{~nm})$ trilayer measured at 300 and $20 \mathrm{~K}$, respectively. about $80 \mathrm{~K}$ and then levels off or even slightly decreases again. In order to understand the different temperature dependence of $J^{(1)}$ and $J^{(2)}$ we note that all MOKE loops taken in the first maximum show remanent magnetization [e.g., Figs. 2(a) and 2(b)], whereas the remanence is zero in the second peak [e.g., Figs. 2(c) and 2(d)]. In Fig. 5 we show the results of simulations for the remanent state, i.e., $H=0$ in Eqs. (2) and (3), for different $J$ values while varying the relative strength of the bilinear and biquadratic coupling, $J_{1} / J_{2}$. Remanence appears when $J_{2}$ dominates over $J_{1}$. A qualitatively similar statement has been given in Ref. [21] for the case $K=0$, where an analytical expression for the dependence of $M_{R}$ on $J_{1}$ and $J_{2}$ is obtained. Thus, the temperature dependence of $J^{(2)}$ is dominated by the bilinear coupling while for $J^{(1)}$ biquadratic coupling is prevailing. Therefore, the different shape of the curves in Fig. 4 reflect different temperature dependencies of $J_{1}$ and $J_{2}$.

The temperature behavior of $J_{2}$ depends on its cause. We exclude intrinsic, higher order contributions [8] as the cause of $J_{2}$ because $J_{2}$ is comparable or even bigger than $J_{1}$. On the other hand, the observed increase of the total coupling $J$ upon cooling is on the order of 3 [Fig. 4]. The loose spins model favored in Ref. [22] predicts a stronger exponential temperature dependence of $J_{2}$ [23]. Hence, it is likely that the big biquadratic contribution is caused by spatial or compositional fluctuations at interfaces [13,21,24]. A more detailed analysis of the origin of the biquadratic coupling is beyond the scope of this Letter. However, for all known biquadratic coupling mechanisms - intrinsic, higher order term [8], loose spins model $[23,25]$, and fluctuation model $[21,24]-J_{2}$ monotonically increases upon cooling and eventually saturates at low temperatures. Therefore, our data indicate that $J_{1}$ levels off below $80 \mathrm{~K}$. However, this shows up only in the total coupling when $J_{1}$ is dominant (curve $J^{(2)}$ in Fig. 4) but is hidden when $J_{2}$ prevails (curve $J^{(1)}$ in Fig. 4).

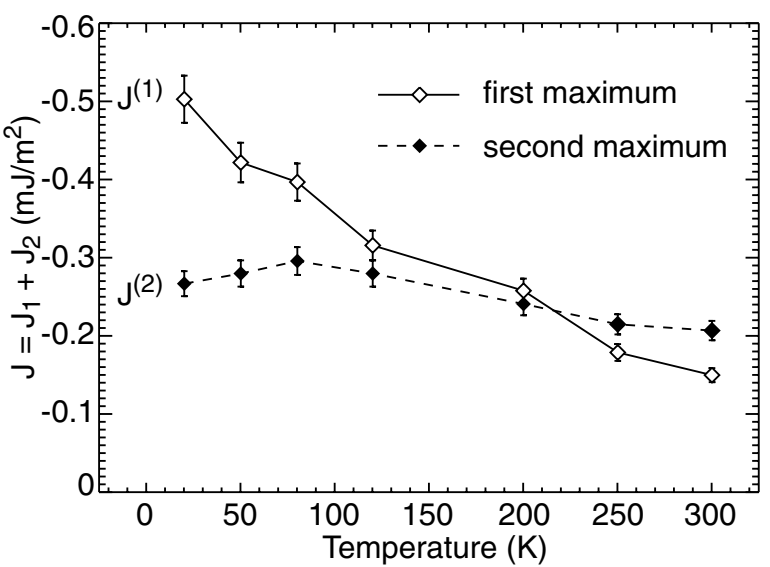

FIG. 4. Total coupling strengths $J^{(1)}$ (open symbols) and $J^{(2)}$ (filled symbols) at the first and second coupling maxima, respectively, as a function of temperature. 


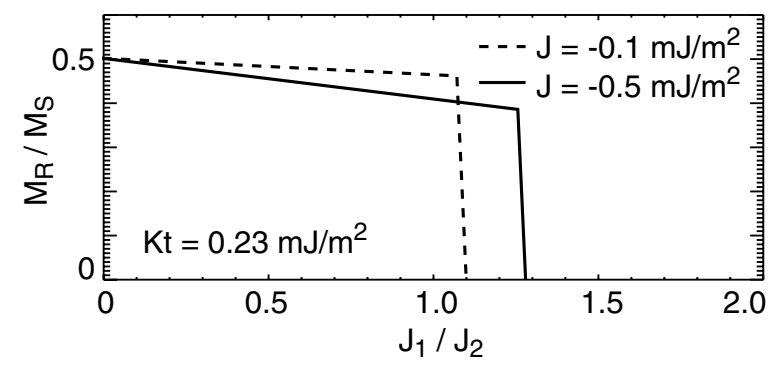

FIG. 5. Dependence of the remanent magnetization $M_{R}$ on the relative strength of the bilinear and biquadratic coupling $J_{1} / J_{2}$ obtained from simulations based on Eqs. (2) and (3) for $H=0$. The anisotropy is kept constant, $K t=0.23 \mathrm{~mJ} / \mathrm{m}^{2}$, and the total coupling $J$ is varied in the range covered in Fig. 4.

The temperature dependence of the bilinear coupling strength for metallic and insulating spacers can be described by the quantum interference model formulated by Bruno [8]. Oscillations and an increase of the coupling strength upon cooling with saturation at low temperatures are found for metallic spacers. In contrast, for insulating spacers the coupling strength is expected to exponentially decay with spacer thickness and to decrease with decreasing temperature. We emphasize that below $80 \mathrm{~K}$ the coupling is also metallic-type, because we observe oscillatory coupling behavior in Fig. 3 down to $20 \mathrm{~K}$.

The temperature dependence of $J_{1}$ might be connected with the resistance behavior of the $\mathrm{FeSi}$ spacer. The temperature-dependent resistance of thin $\mathrm{FeSi}$ films is complex because different crystalline phases with different resistivity behaviors compete: $\mathrm{Fe}_{1-x} \mathrm{Si}_{x}$ with $x \approx 0.5$ grows either in the epitaxially stabilized B2 phase [26] or-depending on thickness and deposition temperature $[7,27]$ - in the bulk stable B20 phase [28]. For instance, a significant reduction of the number of carriers in the spacer near the Fermi level as a function of temperature in the B20 phase could lead to the observed behavior of the bilinear coupling $[8,29]$.

In conclusion, we have demonstrated for the first time oscillatory interlayer coupling across $\mathrm{FeSi}$ spacer layers. The epitaxial growth obtained by coevaporation allowed us to produce samples with superior structural and compositional homogeneity up to $34 \AA$ spacer thickness. The coupling strength increases with decreasing temperatureat least down to $80 \mathrm{~K}$. The temperature dependence, the oscillatory behavior, and the order of magnitude of the coupling strength all imply that the coupling across ordered FeSi can be understood in terms of the conventional models for interlayer coupling across metallic spacer lay- ers without the need to claim a new type of coupling for this specific material.

This work is supported by the HGF-Strategiefondsproject "Magnetoelectronics."

*Author to whom correspondence should be addressed. Electronic address: D.Buergler@fz-juelich.de

[1] P. Grünberg et al., Phys. Rev. Lett. 57, 2442 (1986).

[2] S. S. P. Parkin, Phys. Rev. Lett. 67, 3598 (1991).

[3] S. Toscano, B. Briner, H. Hopster, and M. Landolt, J. Magn. Magn. Mater. 114, L6 (1992).

[4] E. E. Fullerton et al., J. Magn. Magn. Mater. 117, L301 (1992).

[5] A. Chaiken, R. P. Michel, and M. A. Wall, Phys. Rev. B 53, 5518 (1996).

[6] G. J. Strijkers, J. T. Kohlhepp, H. J. M. Swagten, and W. J. M. de Jonge, Phys. Rev. B 60, 9583 (1999).

[7] M. Fanciulli et al., Phys. Rev. B 59, 3675 (1999).

[8] P. Bruno, Phys. Rev. B 52, 411 (1995).

[9] J. J. de Vries et al., Phys. Rev. Lett. 78, 3023 (1997).

[10] Y. Endo, O. Kitakami, and Y. Shimada, Phys. Rev. B 59, 4279 (1999).

[11] R. Kläsges et al., Phys. Rev. B 56, 10801 (1997).

[12] Y. Endo, O. Kitakami, and Y. Shimada, J. Magn. Soc. Jpn. 21, 541 (1997).

[13] M. Schäfer et al., J. Appl. Phys. 77, 6432 (1995).

[14] D. E. Bürgler et al., Surf. Sci. 366, 295 (1996).

[15] D. E. Bürgler et al., Phys. Rev. B 56, 4149 (1997).

[16] M.P. Seah, in Practical Surface Analysis, edited by D. Briggs and M.P. Seah (John Wiley \& Sons, Sussex, England, 1990), Vol. 1, Chap. "Quantification of AES and XPS,” pp. 201-255.

[17] D. Berling et al., J. Magn. Magn. Mater. 191, 331 (1999).

[18] S.-S. Yan, R. Schreiber, P. Grünberg, and R. Schäfer, J. Magn. Magn. Mater. 210, 309 (2000).

[19] M. Goiran et al., J. Magn. Magn. Mater. 172, 199 (1997).

[20] D. E. Bürgler et al., Phys. Rev. Lett. 80, 4983 (1998).

[21] E. E. Fullerton and S.D. Bader, Phys. Rev. B 53, 5112 (1996).

[22] G. J. Strijkers, J. T. Kohlhepp, H. J. M. Swagten, and W. J. M. de Jonge, Phys. Rev. Lett. 84, 1812 (2000).

[23] J. C. Slonczewski, J. Appl. Phys. 73, 5957 (1993).

[24] J. C. Slonczewski, Phys. Rev. Lett. 67, 3172 (1991).

[25] M. Schäfer et al., J. Appl. Phys. 77, 6432 (1995).

[26] E. G. Moroni, W. Wolf, J. Hafner, and R. Podloucky, Phys. Rev. B 59, 12860 (1999).

[27] K. A. Mäder, H. von Känel, A. Baldereschi, Phys. Rev. B 48, 4364 (1993).

[28] Z. Schlesinger et al., Phys. Rev. Lett. 71, 1748 (1993).

[29] Z.-P. Shi, R. P. Singh, and B. M. Klein, Europhys. Lett. 29, 585 (1995). 